\title{
Essais
}

ESSAIS

Revue interdisciplinaire d'Humanités

Résister entre les lignes

\section{Voies et voix de la résistance pendant la dictature en Espagne : Antigone, du mythe à l'incarnation de la dissidence antifranquiste}

\section{Fanny Blin}

\section{(2) OpenEdition}

Journals

Édition électronique

URL : http://journals.openedition.org/essais/4502

DOI : 10.4000/essais.4502

ISSN : 2276-0970

Éditeur

École doctorale Montaigne Humanités

Édition imprimée

Date de publication : 15 juillet 2016

Pagination : 80-94

ISBN : $978-2-9544269-8-3$

ISSN : 2417-4211

Référence électronique

Fanny Blin, « Voies et voix de la résistance pendant la dictature en Espagne : Antigone, du mythe à l'incarnation de la dissidence antifranquiste », Essais [En ligne], 9 | 2016, mis en ligne le 23 octobre 2020, consulté le 29 octobre 2020. URL : http://journals.openedition.org/essais/4502 ; DOI : https:// doi.org/10.4000/essais.4502 


\title{
Voies et voix de la résistance pendant la dictature en Espagne : Antigone, du mythe à l'incarnation de la dissidence antifranquiste
}

\author{
Fanny Blin
}

Parmi les symboles les plus structurants de la notion de résistance politique, Antigone s'est imposée au cours du $\mathrm{XX}^{\mathrm{e}}$ siècle en tant que chantre de l'opposition à la tyrannie, dans l'imaginaire collectif d'une Europe qui voyait ses conflits contemporains se refléter dans la tragédie des Labdacides. Figure mémorielle, emblème de la voix endeuillée, sa lutte pour offrir une digne sépulture à son frère constitue un motif obsédant dans la littérature européenne qui a hérité du mythe grec et de la tragédie de Sophocle ${ }^{1}$. Si l'ouvrage de Steiner ${ }^{2}$ éclaire et historicise l'omniprésence d'Antigone en philosophie comme en littérature, il a contribué à limiter l'analyse aux productions germanophones, anglophones et francophones. Le phénomène de la résurgence d'Antigone dans la dramaturgie espagnole requiert pourtant un décryptage particulier, car son foisonnement de (re)créations propose de nouvelles voies de représentations de la résistance. Correspondant à la période de la dictature franquiste (1939-1975) et à la Transition démocratique, les « Antigones espagnoles $»^{3}$ ont été rédigées dans un rapport étroit avec des revendications politiques ancrées dans ce contexte dictatorial. Une telle concentration n'a pas de quoi surprendre puisque l'héroïne mythique incarne la dissidence, comme le souligne Rose Duroux : "De nos jours, Antigone est considérée comme le symbole de la résistance individuelle à l'arbitraire d'État, au nom d'un devoir supérieur. ${ }^{4}$ En revanche, cette profusion de réécritures ne

1 Le lien entre les tragédies sophocléennes et le théâtre engagé a fait l'objet d'une analyse critique dans la thèse de Djiriga Jean-Michel Dago, La lecture idéologique de Sophocle. Histoire d’un mythe contemporain: le théatre démocratique, Université de la Sorbonne Nouvelle Paris 3, 2013.

2 George Steiner, Les Antigones, Paris, Gallimard, 1986.

3 J. V. Bañuls Oller et P. Crespo Alcalá, Antígona(s): mito y personaje: un recorrido desde los origenes, Bari, Levante, 2008, est le seul ouvrage qui cite presque toutes les « Antigones espagnoles » : ils en décomptent dix-sept au cours de la période qui nous intéresse, auxquelles il faut ajouter les œuvres de Carrión (1940) et de Queizán (1989).

4 Rose Duroux et Stéphanie Urdician, Les Antigones contemporaines (de 1945 à nos jours), Clermont- 
s'accompagne ni d'unité stylistique ni linguistique, ni a fortiori, idéologique. Les dramaturges républicains, en particulier ceux de l'exil, ont emprunté la voix d'Antigone pour porter sur scène un discours critique envers l'exercice tyrannique du pouvoir. Les nombreux parallélismes entre le mythe et l'histoire la guerre d'Espagne constituent un premier ensemble d'éléments expliquant un tel engouement pour l'histoire de la jeune Thébaine. En effet, les mythèmes ${ }^{5}$ [soulèvement interne - lutte fratricide - interdiction d'enterrer les vaincus - répression violente], présents dans Les sept contre Thèbes, Edipe roi

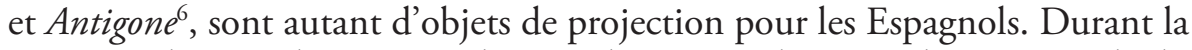
posguerra, les grands récits mythiques alimentent d'un côté la propagande du régime - dans le but de le justifier - et de l'autre, le discours des opposants. L'histoire de cette famille déchirée devient alors la synecdoque idéale pour représenter la nation. Or la guerre pose en soi un problème de représentation, et requiert des contournements, voire des évitements qui sont d'éloquentes béances ${ }^{7}$. Comment surmonter la contradictoire nécessité de dire l'insupportable silence imposé par le régime franquiste sur le sort des vaincus? Comment résister aux entraves du discours ? Les écrivains se trouvent aux prises avec l'objectif paradoxal de mettre le silence en mots, de contourner la censure tout en la bravant. Il s'agit de résister, mais sans risquer d'être privé du lien avec le public, sans quoi l'écriture engagée perdrait tout son sens. Dans un tel contexte, le théâtre se trouve être un moyen d'expression idéal, car les aspects visuels de la mise en scène peuvent pallier les failles expressives du langage. Les dramaturges sont alors confrontés au défi de représenter sur scène une double horreur : celle de la guerre, mais aussi celle de ne pas pouvoir en parler. C'est de la tension entre ces deux nécessités qu'est né un discours de résistance à proprement parler. Celui-ci a été porté par des auteurs qui ont transcendé l'indicible en s'emparant d'une figure mythique de l'insoumission : Antigone. Réécrire une tragédie qui a traversé les siècles suppose un détour par un discours qui préexiste, déjà chargé de symboles. Beaucoup d'Antigones espagnoles furent des paraboles à la fois dénonciatrices et protectrices. Par conséquent, elle s'avère un outil précieux pour résister à l'univocité du discours officiel sur l'histoire nationale, prenant une voie et une voix alternatives. Cette réflexion s'attèlera à mettre en lumière les dispositifs employés

Ferrand, Presses Universitaires Blaise Pascal, 2010, introduction, «Antigone. Retours sur une fascination ", p. 18.

5 Développé par Claude Lévi-Strauss à partir du mythe d'CEdipe, le concept renvoie aux unités irréductibles de la structure "narrative " du mythe. Voir " La structure des mythes ", Anthropologie structurale I, Paris, Plon, 1958.

6 Eschyle, Les sept contre Thèbes, 467 av. J.-C. ; Sophocle, Antigone, 441 av. J.-C. ; Sophocle, Edipe roi, 430-420 av. J.-C.

7 La désignation même pose problème, puisque le gouvernement militaire interdit aux citoyens de parler de guerre civile, imposant la formule « Glorioso Alzamiento nacional». Sa représentation se trouve donc d'autant plus perturbée que le nom de l'épisode historique est sujet à manipulations. 
par les dramaturges espagnols qui ont repris ce personnage emblématique afin d'apposer une pensée et une parole sur leur histoire, qu'ils ont projetée sur la scène théâtrale, en tant que miroir déformant de la société.

\section{La tragédie, cadre structurant de la représentation de la résistance}

Représenter la résistance, convertie en objet de fiction, implique une conceptualisation préalable, qui va de pair avec une prise de distance. Cela suppose en effet de passer à l'ordre du symbole pour la figurer. Le choix d'une figure issue de la mythologie grecque incarnant la ténacité s'avère alors probant. Cela constitue un détour : l'incarnation du concept pallie la difficulté, voire l'impossibilité ${ }^{8}$ de l'exprimer. À ce sujet, une définition du mythe synthétise le sens d'une telle résurgence : à propos de Cassandre - autre figure de la transmission mémorielle - il était souligné que le mythe résiste intrinsèquement au temps :

"Par ses capacités d'ouverture et de résistance, le mythe est un matériau apte à transmettre une mémoire et à la renouveler. Outil de symbolisation, il est un scénario que l'on peut faire sien, réinvestir, pour exprimer une expérience personnelle ou collective majeure, parfois traumatique voire indicible. »"

Les penseurs espagnols se saisissent de la figure d'Antigone pour incarner, au sens fort du terme, un concept de résistance qui semble central et pourtant évanescent à cette période. La notion même est donc interrogée et déconstruite à travers sa mise en scène, aboutissant à l'incarnation de la pensée en action. Ce processus suppose la démythification de l'argument tragique, et implique une perspective ontologique, à l'instar de l'appropriation de la figure antigonienne par María Zambrano. Celle-ci avait écrit dans un premier temps un "délire d'Antigone ${ }^{10}$, or le terme de délire illustre à la perfection le fil tortueux d'une réflexion existentielle chez l'héroïne en crise. Cette œuvre donne à penser l'avènement de la conscience individuelle et le rapport de l'individu au pouvoir autoritaire. Au cœur des réécritures par les exilés républicains, se lit une réflexion sur le sens de la résistance qui s'inscrit dans une dynamique autofictionnelle. José Bergamín, par exemple, accentue le rapport étroit entre le personnage d'Antigone et l'errance, rappelant maintes fois qu'elle a accompagné son père Edipe dans son destierro ${ }^{11}$, ce qui fait d'elle un

8 Cet indicible relève à la fois du trauma, de l'auto-censure et de la censure officielle.

9 Appel à contributions (2013) Cassandre, figure du témoignage et de la transmission mémorielle, Kimé, coll. "Entre Histoire et mémoire ", Véronique Léonard-Roques et Philippe Mesnard (éd.), Clermont-Ferrand, Université Blaise Pascal, 2015, à paraître.

10 M. Zambrano, "Delirio de Antígona ", Orígenes, La Havane, n 18, 1948, p. 14-21. La notion de délire constitue un des points d'achoppement de la pensée zambranienne. Cf. María Zambrano, Delirio y destino, Madrid, Centro de Estudios Ramón Areces, 1988.

11 Le Coryphée chante la douleur de l'exil, avec le champ lexical du conflit espagnol : le retour 
guide, un personnage lumineux porteur d'espoir dans les ténèbres de l'exil. Cette dimension vaut autant pour la fiction que dans le cadre de l'expérience personnelle du poète, qui se sauve en s'appropriant une telle figure. Dans cette pièce, Tirésias met en garde contre la condamnation de la jeune femme, et Bergamín lui prête ces mots : "Antigone ne doit pas mourir. " ${ }^{12}$ Dans cette scène, le devin sort du champ de l'action dramatique pour s'élever au-dessus de la fiction afin d'en analyser les symboles. Communiquant plus " directement " avec le public, sur un terrain hybride entre contexte espagnol et postérité du mythe, Bergamín brise le quatrième mur, afin de forcer l'adhésion. Tirésias incite chacun à prendre du recul sur le sort d'Antigone, non plus en tant que personnage du drame, mais en tant que figure de la résistance. Car « Antigone ne doit pas mourir "signifie bien que sa parole ne peut pas s'arrêter avec la mort. Son message, transmis aux spectateurs, devient immortel : le texte fait d'eux les héritiers de son rôle, les met sur la voie d'une réflexion métahistorique qui les encourage à reprendre à leur compte la résistance de l'héroïne. Zambrano et Bergamín mènent à travers cette figure un cheminement esthético-philosophique, fondé sur l'incarnation. Camile Lacau St Guily le signale : "Lorsque l'Antigone de Zambrano dit non, c'est précisément parce qu'elle représente une forme de résistance "dramatique". Elle est une résistante, l'incarnation d'une philosophie vivante, puissante. ${ }^{13}$ La représentation de leur douloureuse condition d'exilés repose sur la métaphore du chemin, motif obsédant dans la majorité des réécritures, qui symbolise lui-même le destin funeste. Ces détours métaphoriques sont autant de voies pour dire l'indicible, de façon détournée, avec des jeux d'opposition, une polysémie, des clins d'œil au lecteur sur les références au contexte, et créent un code qui instaure une complicité du public dans la résistance, requérant une lecture active qui force le parti-pris. Antigone prête ainsi sa voix aux résistants tout en devenant le masque qui les protège de la répression et parfois, de la censure.

\section{La reprise de ce mythe constitue d'emblée un acte ostensiblement engagé}

La subversion se doit de résister à la censure. Or durant la première période du franquisme les publications ont été régies par la Ley de Prensa de 1938, une loi de guerre visant à enrayer les canaux de diffusion de la République. Le texte établi par Serrano Suñer établissait la censure pour les écrits qui sème-

possible des exilés « cuando tornamos del destierro " (Bergamín, La sangre de Antígona, p. 383). "Apenas han vuelto y ya mueren". Bergamín introduit une référence aux exilés dont le retour est souvent amer au début de la Transition.

12 "Si condenáis a Antígona a morir destruís la paz de la vida para el hombre. ¿Por qué separáis a unos de otros como si fuera lo justo? ¿Por qué?». Tirésias dépeint donc Antigone comme le symbole de la paix, et implore Créon de l'épargner : “¿Que no muera Antígona!» (p. 414).

13 C. Lacau St Guily, María Zambrano, La tumba de Antígona, Paris, PUF, CNED, 2013. 
raient des "idées pernicieuses " trop critiques envers le gouvernement ${ }^{14}$, et sanctionnait la "résistance passive et en général, le fait de dévier des normes dictées ${ }^{15}$. Or cette loi issue de l'état d'exception a perduré pendant près de trente ans, restant en vigueur jusqu'à l'instauration de la Ley de Prensa de 1966. Jusqu'en 1951, le "Servicio de Inspección de Libros " appliquait la Loi de 1938 pour la censure des ouvrages de fiction, après quoi le Ministerio de Información y Turismo a été créé dans le but gommer la " mauvaise image qu'offrait une censure aussi arbitraire. " ${ }^{16}$ Si la Ley Fraga visait à en finir avec le contrôle total des productions, "la libertad de expresión no se normalizó y la censura persistió. ${ }^{17}$ Le MIT se réservait le droit de supprimer ce qui ne plairait pas aux censeurs et celui de déconseiller la publication de passages ou encore celui d'interdire l'édition. La Ley Fraga n’a donc pas libéré le discours sur les sujets sensibles comme la guerre civile, et les références à l'armée, à la religion, aux grèves, à la résistance, qui pouvaient insuffler des idées " dangereuses ". Dans ces conditions, l'appropriation du mythe fonctionne comme un outil de dénonciation déguisée, protégée par le prétexte de réécrire un classique. Mais ce n'est pas là le seul détour auquel se livrent les dramaturges engagés.

\section{Antigone, masque antique de la résistance contemporaine}

Le mythe constitue en effet un " écran de projections " pour les résistants. Or Antigone renaît, trouve sa voie et sa voix par la parole, ce qui est une claire métaphore de la réécriture. Zambrano vit précisément une forme de renaissance par la réécriture, et son personnage s’impose de la même manière que la philosophe elle-même n'existe - en tant que résistante - que lorsqu'elle s'exprime. Le même parallélisme entre dramaturge et personnage s'illustre dans l'œuvre La raison d'Antigone ${ }^{18}$, de Carlos de la Rica, écrite pendant la dictature, qui est dédiée " aux peuples opprimés et à tous ceux qui luttent pour la liberté et la démocratie. " ${ }^{19}$ Le personnage de Créon y campe un avatar à peine déguisé de Franco, dont le pouvoir émane d'une guerre fratricide. L'action dramatique a lieu dans un théâtre, jusqu'à l'arrestation de l'auteur par la police, qui intervient pour annoncer que le pouvoir a censuré ses écrits. Le métalangage théâtral et cette mise en abyme permettent d'assumer un discours politiquement engagé

14 Ley de Prensa du 22/04/1938 : "Artículo décimo octavo: [...] castigar gubernativamente todo escrito que directa o indirectamente tienda a mermar el prestigio de la Nación o del Régimen, [...] o siembre ideas perniciosas entre los intelectualmente débiles. "

15 Ibid., "Artículo décimo noveno: También serán sancionadas las faltas de desobediencia, resistencia pasiva [...] las de desvío a las normas dictadas [...]».

16 Cisquella, Erviti et Sorolla, La represión cultural en el franquismo: diez años de censura de libros durante la Ley de Prensa, 1966-1976, Anagrama, 2002, p. 23.

17 Ibid, p. 27.

18 C. De la Rica, La razón de Antígona, Cuenca, El Toro de Barro, 1980.

19 "A los pueblos oprimidos y a cuantos luchan por la libertad y la democracia", ibid. 
et risqué, au même titre que celui d'Antigone, qui risque sa vie. Le fait que les dramaturges se mettent eux-mêmes en scène dans un rôle subversif et soumis à la censure constitue une représentation par excellence de leur condition de résistants. Au-delà du reflet que crée la mise en scène des répétitions de la pièce, le miroir doit être renvoyé au public : son double héroïque possible apparaît alors sur scène, qui permet que le public s'identifie. Afin de créer une action exemplaire, voire cathartique, les dramaturges soulignent le lien entre le mythe et l'actualité et soulignent la légitimité de la résistance. Pour ce faire, l'action est souvent projetée dans un contexte familier pour le spectateur afin de renforcer la similitude avec la situation de l'héroïne. Tout l'enjeu est de révéler cette légitimité sur scène, en valorisant la protagoniste. Michel Richard écrit au sujet de la désobéissance : "Face à une dictature ou à une dérive autoritaire, la résistance à l'oppression et plus encore l'insurrection sont risquées, mais légitimes. ${ }^{20} \mathrm{Il}$ s'agit de dénoncer la dérive autoritariste en renversant le rapport entre loi et justice immanente. Sur scène, il est ostensiblement montré que la citoyenneté exemplaire est dans le camp de la désobéissance. Cette réflexion menée à travers les réécritures s'inscrit dans un contexte qui obligeait à repenser la notion de résistance, distinguant légalité et légitimité.

\section{Contournements de représentation : une parole oblique contre le silence imposé}

Le détour le plus récurrent consiste en une subtile caricature du tyran à travers la ridiculisation du personnage de Créon, caractérisé par son hybris. C'est à travers ce filtre que les stratégies mises en œuvre par Franco pour briser toute résistance sont caricaturées dans les pièces, fomentant la métatextualité autant que la mise en abyme de la situation d'écriture entravée. En premier lieu, il est montré que le pouvoir est aveugle, puisqu'il est incarné par un tyran antipathique, borné et fou. La violence de Créon est mise en avant dans la pièce de Rodríguez Pampín, à travers la mise en scène de sa folie meurtrière. Illustrant les travers de l'hybris, la violence de Créon trouve ses racines dans son refus de voir s'effondrer sa logique : il nie la parole d'Antigone jusqu'à vouloir la faire taire, la priver de son moyen d'expression, et de son existence même. Il répète que les morts vont disparaître, qu'il " n'en restera aucune trace ", ce qui fait allusion à la problématique de mémoire officielle des faits de la guerre civile. Créon : "Mortos, mortos, todos mortos! (totalmente fora de si) E non quedará rastro. ${ }^{21}$ Il finit par étrangler de ses propres mains sa nièce Antigone. Il est très significatif de constater que Pampín se livre à une mise

20 M. Richard, «La désobéissance », in Gilles Ferréol (éd.), Rapport à autrui et personne citoyenne, Poitiers, CRDP, 1998, p. 172-178.

21 X. M. Rodríguez Pampín, Creón... Creón, Grial, n 50, 1975, p. 492 : « Morts, ils seront tous morts! (complètement hors de lui). Et il n'en restera aucune trace. " (Toutes les traductions sont de l'auteur) 
en scène hautement symbolique de la répression envers la résistance, qui dévie radicalement de Sophocle. Dans cette pièce de 1975, la mort de l'héroïne n'est plus cachée, mais représentée : elle a lieu sous les yeux du public, qui assiste à cet ostensible excès du tyran et à l'extinction de la voix de cette figure, par étranglement ${ }^{22}$. Elle s'écroule sur scène en tant que porte-drapeau de la résistance, plaignant son oncle de l'extrémité à laquelle il est parvenu.

La folie de la dictature se représente à travers la sentence réservée à Polynice, qui sert d'exemple afin de dissuader les velléités de résistance. Mais le dictateur est également moqué à travers sa compromission : il est parfois représenté comme doucereux, essayant de persuader Antigone de se rendre et de renier son acte. Il lui propose clairement un traitement de faveur et met en péril l'intégrité de son pouvoir en se dédisant. La dénonciation et la caricature passent par la mise en scène des coulisses du pouvoir. À travers un jeu sur le rapport entre ce qui est caché et montré, les réécritures inversent la logique spatiale entre coulisses et scène. En fait c'est à la fois son inflexibilité (comme chez Espriu par exemple) et sa versatilité (comme chez Rodríguez Pampín) qui sont critiquées. Au sujet du tyran de la version galicienne, María Margarita Doncel remarque : "Sobresale la denuncia tajante contra el poder inflexible y tiránico de los déspotas. La transgresora Antigona se convierte en simbolo de los ideales humanos de fraternidad, paz y libertad en contraposición a la opresión politica. " ${ }^{23}$ L'aspect grotesque du pouvoir est également souligné chez Elizondo ${ }^{24}$, qui représente une pantomime ridicule dans la prison où se déroule l'action, montrant que Créon n'est qu'un simple pantin. De la Rica également dénonce la pantomime du pouvoir : Créon n'est qu'un pantin sénile manipulé par ses conseillers - à l'image de Franco ? - : "Contemos incluso, con la debilidad senil de Creón. " 25 On assiste dans cette scène à la révélation de la compromission des gouvernants et leurs intentions de manipulation du peuple : "Permitamos algunos desordenes. Pero tendremos la confianza si sabemos engañar al pueblo porque el país sólo desea tranquilidad ${ }^{26}$. Par son écriture démythifiante, Riaza fait dire à son Créon : " no soy

22 Cette mise en scène de l'assassinat s'écarte du schéma tragique traditionnel où le suicide d'Antigone n'est pas représenté mais constaté par Hémon. Ce meurtre en direct élève le spectateur au statut de témoin des exactions de la dictature. La résistante meurt sans procès ni condamnation et Créon exécute lui-même ce meurtre, étouffant sa nièce et la contestation.

23 M. M. Doncel, "De Sófocles a Luis Rafael Sánchez y Otras Antígonas ", Inter-Ethica, ("Universidad Interamericana de Puerto Rico, Ponce ") : "La dénonciation sans pitié est évidente, envers le pouvoir inflexible et tyrannique des despotes. La transgressive Antigone devient le symbole des idéaux humains de fraternité, paix et liberté, qui s'opposent à l'oppression politique.".

24 J. Martín Elizondo, Antígona entre muros, Primer acto, n 329, p. 145-190.

25 C. De la Rica, op. cit., p. 61 : « Nous comptons également sur la faiblesse sénile de Créon. »

26 Ibid., p. 61 : «Permettons quelques troubles. Mais nous gagnerons la confiance si nous savons duper le peuple car le pays ne souhaite que la tranquillité. " 
ese personaje inflexible que imaginas. " ${ }^{27}$ L'usage du terme " personnage " invite le lecteur à s'interroger sur la vérité dans le cadre politique, mais également sur la fidélité de la réécriture. Créon tel qu'on l'attend - inflexible - n'est qu'un personnage tragique : selon le propre personnage de Riaza, il est différent de sa version contemporaine, qui verse dans la compromission.

\section{Scénographie de la désobéissance}

Dans ces réécritures, l'acte de désobéissance d'Antigone la définit comme une opposante : tout bascule lorsqu'elle décide de désobéir. Or l'analyse des textes dramatiques révèle qu'il ne suffit pas de désobéir pour résister, mais que l'enjeu crucial est le discours de résistance. La preuve : Créon, dans certaines versions, tente de négocier avec Antigone, lui proposant de passer son acte sous silence si elle accepte de garder le secret. Ce n'est donc pas le fait qu'elle ait bravé le dictateur en allant enterrer son frère qui la définit comme résistante, c'est le fait de continuer coûte que coûte à l'assumer. Dans la pièce de Rodríguez Pampín, Antigone clame sa fierté d'avoir apporté des fleurs sur les tombes de ses frères, en dépit des ordres de Créon, qui, comme nous le commentions, ne peut supporter cet affront et - de rage - l'étrangle. Cette mise à mort par étouffement montre bien que le plus important n'est pas qu'elle ait désobéit mais qu'elle le dise. Il faut la faire taire et poser une chape sur la résistance. La notion de censure de la parole est ainsi omniprésente dans les textes. Par exemple, Espriu fait dire à son Créon : "Debo ordenar crueles leyes que mantengan en silencio los labios del vencido. ${ }^{28}$ Quant à Bergamín, il écrit alors en tant qu'artiste dont la voix exilée ne pouvait pas atteindre le public espagnol de l'intérieur. La supplique d'Hémon qui implore Antigone de ne pas se taire montre que mourir c'est cesser de parler, et donc de se rebeller. Bergamín faire dire à Hémon : " ¡Aunque no pueda llegarnos tu voz, háblanos! ${ }^{29}$. Il semble presque s'agir d'un manifeste visant à convaincre les exilés de l'intérêt de résister par la parole.

Les Antigones espagnoles transforment la scène théâtrale en terrain de lutte politique. Le dilemme entre résister et céder est incarné par les deux sœurs Ismène et Antigone, qui ne prendront pas le même chemin. Pendant la dictature, une Antigone a émané du Théâtre indépendant : celle de M. Bayo et J. Sanchis Sinisterra, intitulée Ahora en Tebas ${ }^{30}$. Les Grupos de Teatro Universitario étaient alors des foyers culturels de résistance idéologique. Malgré leur ados-

27 L. Riaza, Antígona ... jcerda!, Mazurka, Epilogo, Madrid, La Avispa, 1983, p. 271 : « je ne suis pas ce personnage inflexible que tu imagines. "

28 S. Espriu, Antígona, Palma de Mallorca, Moll, 1955: « Je dois dicter des lois cruelles qui maintiennent en silence les lèvres du vaincu. »

29 J. Bergamín, op. cit., p. 422 : " même si ta voix ne nous arrive pas, parle-nous!"

30 M. Bayo (en tant qu'écrivain) et J. Sanchis Sinisterra (qui en fut le théoricien et metteur en scène) ont conçu ensemble Ahora en Tebas, inédite, jouée en 1963. 
sement au syndicat étudiant unique, ils parvenaient à rester en marge et à mettre en scène des œuvres assez libres, devant déjouer la censure avec des double-sens. José Sanchis témoigne ${ }^{31}$ que la clôture de l'œuvre "Qué fácil es la paz " ${ }^{32}$, renvoyant avec provocation à la célébration de plus de "veinte años de paz ", leur avait fait craindre d'être censurés, d'autant que l'ambiguïté régnait sur les règles de la censure. Reprendre Antigone était en soi un acte de résistance, à l'image de cette réplique d'Antigone, qui clame sa désobéissance devant le peuple : "Y yo digo a los que mandan en la ciudad que yo le sepultaré. ${ }^{33}$ De plus, elle fait cette déclaration de sécession là où le peuple se réunit (forme d'agora) ce qui n'est pas anodin : le parcours d'Antigone commence là où Tirésias vient d'appeler le peuple à résister ${ }^{34}$ à la tyrannie de Créon. De la même manière, réécrire cette histoire dans une langue comme le catalan ou le galicien, comme l'ont fait une dizaine de dramaturges pendant la dictature et la Transition, prend un sens tout particulier : il s'agit de représenter le concept dans une langue dont l'usage est en soi une résistance.

\section{Résister à l'univocité linguistique et historiographique}

Pour les dramaturges qui écrivent en Espagne dans une autre langue que le castillan, il s'agit de résister à l'uniformisation culturelle du Régime. La résistance des artistes "de l'intérieur " s'exprime parfois à travers l'écriture dans une langue proscrite. Or, emmener Antigone en Catalogne ou en Galice constitue en soi un acte de revendication, par rapport à la politique linguistique du Régime. "La censura fue especialmente dura con cualquier libro escrito en cualquiera de los tres idiomas que coexisten con el castellano en España. Y ello fue particularmente cierto en el caso de la lengua catalana, mucho más presente en la producción cultural que el gallego o el euskera. " ${ }^{35}$. Par exemple, la version de Salvador Espriu ${ }^{36}$, écrite en 1939 dans une Barcelone occupée par les troupes nationalistes, est restée inédite jusqu'en 1958. Ces réécritures participent de la construction d'une mémoire collective alternative, dans un véhicule linguistique alternatif, et contribuent également à ce que R. Duroux appelle les "Mémoires autonomiques " ${ }^{37}$. La politique de mémoire étant entravée

31 Entretien mené le 12/05/15, à Madrid.

32 M. Bayo, Ahora en Tebas, manuscrit inédit, p. 50.

33 Ibid., p. 36 : " et moi je dis à ceux qui commandent dans la cité que je l'enterrerai."

34 Ibid., p. 36 : Tirésias : "¿Hasta cuando habrá que esperar y sufrir para que seáis Tebas?", "Jusqu’à quand faudra-t-il attendre pour que vous soyez Thèbes? ". Le dramaturge insuffle à sa pièce la révolte qui se fait sentir dans les années 1960 : cette harangue de Tirésias s'adresse directement aux Espagnols.

35 Cisquella, Erviti et Sorolla, op. cit.

36 S. Espriu, op. cit.

37 R. Duroux, "La "juste mémoire" ?", Cahiers de civilisation espagnole contemporaine. De 1808 au temps présent, $\mathrm{n}^{\circ} 2,2008$, p. 9. 
et disparate, la fiction permet de combler, et parfois même " d'inventer " un récit de glorieuse résistance. L'écriture se fait alors performative afin de « fabriquer » un passé de résistance. Cependant, il faut insister sur le fait que toutes les pièces écrites en catalan et en galicien ne sont pas des pièces engagées, et qu'il y a une grande diversité des postulats éthiques d'écriture : il s'agit parfois d'Antigones christiques, symboliques, pas forcément ancrées dans un contexte de contestation politique.

À mesure que le régime militaire se consolide et reste inflexible sur le maintien de l'ordre et la répression, il devient de plus en plus évident, faute de pouvoir mener à bien l'opposition sur le terrain politique, que la littérature occupe une place fondamentale dans les stratégies de diffusion de discours subversifs et autres alternatives à la version de l'histoire imposée par la propagande phalangiste. Or, "La littérature peut jouer un rôle crucial de médiateur entre les mémoires vives des individus et la mémoire des collectivités. ${ }^{38}$ Zambrano invente ainsi un dialogue post-mortem entre les frères ennemis, au cours duquel Polynice se distingue de son frère en dénonçant les faux-semblants de la version officielle du conflit : "el orden que él dice con el orden de la verdad no tiene nada que ver. Se trata solamente de que no salten ciertas verdades. ${ }^{39} \mathrm{Il}$ s'agit là de résister - par la parole - à une vérité officielle qui censure les discours transgressifs : la résistance se joue dans la fiction mais aussi au niveau de l'écriture, car les deux reflètent la nécessité de dire.

\begin{abstract}
"Certes, cette mémoire obsédante n'offrait qu'une seule version du conflit et rejetait dans l'exil les autres interprétations. [...] Quant aux mémoires des vaincus, elles ont été confinées par la répression dans les cercles étroits des souvenirs individuels ou familiaux. Leur fragmentation empêcha longtemps d'atteindre le degré de cohérence narrative nécessaire à leur projection dans l'espace public. ${ }^{40}$
\end{abstract}

La question d'un discours sur la guerre entraîne une guerre des discours, voire une " contre-résistance ". Un des piliers de la politique culturelle du franquisme, José María Pemán, s'est emparé de cette tragédie pour écrire une Antigone qu'on pourrait qualifier en effet de " contre-résistante ». Le cas de cette Antigone est très intéressant parce qu'elle s'inscrit en rupture avec toutes les autres sur le plan idéologique. Pemán n’a pas laissé les dissidents accaparer la figure, et a imposé son " adaptation très libre de la tragédie de Sophocle » ${ }^{41}$. Sa version justifie l'action menée par le Régime en comparant le soulèvement

38 Interférences littéraires, Nouvelle série, $\mathrm{n}^{\circ} 3$, «Les écrivains et le discours de la guerre », FrançoisXavier Lavenne et Oliver Odaert (éd.), novembre 2009, p. 217-225.

39 M. Zambrano, La tumba de Antígona, Madrid, Cátedra, 2012, p. 213 : "L'ordre dont il parle n'a rien à voir avec l'ordre de la vérité. Il s'agit seulement que certaines vérités n'éclatent pas ».

40 S. Michonneau, "Espagne. Les fantômes de la guerre civile et du franquisme », Grande Europe $\mathrm{n}^{\circ}$ 6, mars 2009, La Documentation française, DILA.

41 J. M. Pemán, Antígona, adaptación muy libre de la tragedia de Sófocles, Madrid, Arbor, 1946. 
militaire avec les actes héroïques de l'antiquité. La victoire est due à la volonté divine : "los dioses decidieron esta victoria " ${ }^{42}$, et présentée comme une garantie de liberté dans le futur : "para alumbrar el gozo de nuestra libertad " ${ }^{43}$. Pemán intègre le champ de bataille idéologique en rupture franche avec le discours des vaincus, dont la figure par excellence est dévoyée. Si cela peut sembler paradoxal, Juan Torres ${ }^{44}$ indique que c'est en déplaçant le débat politique sur le terrain religieux que Pemán est parvenu à faire d'Antigone une icône franquiste. Dans le cadre d'un pouvoir qui remédiait à sa fragile légitimité en s'adossant à l'Église catholique ${ }^{45}$, le propos de l'héroïne peut être relu au prisme de la supériorité de la loi divine sur les lois civiles, qui structure la tragédie de Sophocle. Cette œuvre illustre les manipulations mémorielles auxquelles les idéologues phalangistes se sont livrés. Torres rappelle que la pièce de Pemán était diffusée par l'école, autre canal de conditionnement de la pensée et pilier de l'idéologie franquiste : cette Antígona était donc la seule connue de la majorité des citoyens.

Hay otras Antígonas... curiosas, o directamente insultantes. Por ejemplo, la de José María Pemán, el poeta franquista por excelencia, que tuvo la desfachatez de escribir una versión libre de la de Sófocles [...] cuyos fragmentos su amigo Gonzalo Torrente Ballester incluyó en los libros de Formación del Espíritu Nacional que estudiábamos en los colegios. Qué desvergüenza, dirán ustedes. La heroína utilizada por los fascistas, quién puede explicar esto... Bueno, había una causa: los dioses. [...] ¿Qué leyes hay que obedecer en caso de conflicto, las de los dioses o las de los hombres? Es claro, Pemán y el Torrente falangista, y Sófocles también, esgrimen la sabiduría divina como máximo argumento de autoridad. A Polinices había que enterrarlo porque de lo contrario su alma vagaría durante toda la eternidad sin hallar reposo (o, dicho en lenguaje nacional-católico, no hay otra ley que la que emana de la Iglesia Católica Apostólica y Romana). ${ }^{46}$

42 Ibid., p. 63

43 Ibid., p. 61.

44 J. Torres, "Antígona y sus hermanos ", Voz Populi, 15 février 2013, http://vozpopuli.com/ blogs/2196-juan-torres-antigona-y-sus-hermanos.

45 Voir D. Rozenberg, «L'État et les minorités religieuses en Espagne (du national-catholicisme à la construction démocratique) ", Archives des sciences sociales des religions, nº 98, 1997, p. 9-30.

46 J. Torres, art. cit. : "Il y a d'autres Antigones... étranges, ou directement insultantes. Par exemple, celle de José María Pemán, le poète franquiste par excellence, qui a osé écrire une version libre de celle de Sophocle [...], dont certains extraits ont été inclus par son ami Torrente Ballester dans les manuels de Formación del Espiritu Nacional (Formation du Sentiment National) que nous étudions à l'école. Quel culot, direz-vous. L'héroïne utilisée par les fascistes, qui pourrait l'expliquer... Eh bien, il y avait une raison : les dieux. À quelles lois faut-il obéir en cas de conflit, celles des dieux ou celles des hommes ? Il est clair que Pemán, et le Torrente phalangiste, et Sophocle également, brandissent la sagesse divine comme argument d'autorité suprême. Il fallait enterrer Polynice sans quoi son âme errerait pour l'éternité sans trouver le repos (ou, autrement dit en langage national-catholique, il n'y a pas d'autre loi que celle qui émane de l'Église Catholique Apostolique romaine. ». 
Hormis celle de Pemán, la plupart des œuvres véhiculent un message subversif qui dénonce. Elles représentent les travers d'un pouvoir tyrannique qui veut briser à tout prix toute contestation, quitte à faire preuve d'un excès incommensurable, ainsi métaphorisé chez De la Rica : "Creón está borracho de poder y ordena, ordena. $"{ }^{47}$ Mais le message résiste-t-il à toutes les subversions ? Représenter la résistance relève en soi de la lutte pour imposer une voix. Le palimpseste collectif créé autour de cette tragédie contribue à " fabriquer » un passé glorieux, dans une perspective d'écriture performative. C'est pourquoi elle cristallise la figuration de l'opposition, d'autant plus fortement que son caractère théâtral favorise la mise en scène visuelle et sonore d'un conflit marqué par l'indicible, l'inmontrable.

\section{De la résistance active par l'écriture à la pensée critique de la résistance}

Pendant la Transition, le désenchantement aboutit à une critique des anciens résistants. Une des réécritures les plus novatrices est celle de Riaza, qui pousse la démythification à son paroxysme. S'éloignant fortement de la trame tragique, le grotesque sert une critique qui ne se limite pas au tyran et dessine le portrait sans complaisance du renoncement d'Antigone, ancienne féministe hippy qui a bafoué ses idéaux. Son essence résistante est effacée : elle est devenue consensuelle et bourgeoise. Le message de Riaza est celui d'une insurrection contre les anciens résistants, et c'est dans cette mesure qu'il est paradoxalement fidèle à l'hypotexte ${ }^{48}$. D'ailleurs, la critique Aliette Armel le reconnait : "Antigone (...) résiste décidément à tous les mauvais traitements $»^{49}$. À travers l'usage de ce terme de "résister » elle montre que le message reste. D'ailleurs, lui infliger une ultime subversion n'est pas un " mauvais " traitement, puisque c'est là son sens profond. Rodríguez Pampín adopte des techniques encore plus ironiques : il aborde la résistance en représentant sa cruelle absence. Le ton y est désenchanté : le pouvoir veut maintenir à tout prix les faux semblants d'une société où personne n'a résisté. Le personnage du narrateur le souligne en s'adressant au public : «De seguro que todos vos estaredes vendo a Antígona, aproveitando a escuridade e o pouco trafico da noite, correr deica o cementerio e deixar unha frol na campa de Polinice. Pois non. Antigona durme coma todos $»^{50}$. À ce moment, la figure de la résistance est donc niée au

47 C. De la Rica, op. cit., p. 36 : «Créon est ivre de pouvoir, et il ordonne, il ordonne. »

48 Sur la résistance du mythe dans la littérature contemporaine, voir P. Brunel, Mythocritique, Paris, PUF, 1992.

49 A. Armel, (éd.), Antigone, Paris, Autrement, Figures mythiques, 1999.

50 X. M. Rodríguez Pampín, op. cit., p. 481 : «Vous imaginez sûrement Antigone, profitant de l'obscurité, (...) courir jusqu'au cimetière et déposer une fleur sur la tombe de Polynice. Mais non. Antigone dort comme tout le monde. " 
cœur même de sa fonction dans l'imaginaire collectif. Antigone l'ensevelisseuse, supposée vouloir enterrer son frère coûte que coûte, ne quittera pas son confort... Dans cette œuvre précise, la démythification touche non seulement le pouvoir mais aussi ses opposants, elle dénonce les lâchetés en démantelant le symbole même de la rébellion.

En définitive, comment résister à la reproduction du schéma tragique ? La lecture comparée de ces pièces invite à une réflexion métalittéraire sur les enjeux d'une représentation renouvelée de la résistance, au $\mathrm{XX}^{\mathrm{e}}$ siècle. Comment résister à la reproduction du même schéma tragique ? Faut-il défier Sophocle et rompre le modèle ? Cela implique de s'opposer à lui en tant qu'autorité littéraire : c'est ce qu'a osé Zambrano, qui dénonce une faille dans le texte antique. Selon elle le tragédien s'est trompé en représentant la mort d'Antigone par suicide, et elle parle de "l'erreur de Sophocle " ${ }^{11}$, remettant en cause l'autorité suprême en la matière. Elle assume ainsi de provoquer le père symbolique du texte, comme Antigone a défié son oncle. Les parallélismes entre auteur et personnage ne s'arrêtent pas là : l'idée d'erreur - qui renvoie à l'inceste - réapparait lorsqu'Antigone dit : "Hija soy del error " ${ }^{52}$. Or Antigone, en tant que réécriture, naît aussi d'une divergence, d'une réinterprétation sujette à l'erreur. Cette notion révèle la possibilité pour ceux qui s'emparent du mythe de résister aux schémas de l'hypotexte. D'ailleurs c'est justement ce qui fait qu'Antigone "résiste " au temps, en tant que tragédie universelle, grâce aux incartades de ceux qui la réécrivent au présent.

Ces réécritures illustrent chacune à leur manière l'irrésistible nécessité de la résistance. Ils la représentent via le détour par une figure symbolique, qui montre la voie, et se fait le porte-voix de messages contestataires. Si la catharsis opère dans ces pièces de la dictature, Antigone sert alors d'exemple, et ce qu'elle vit sur scène montre qu'il est impossible de ne pas résister. La démonstration est visuelle, incarnée par cette représentation schématique du conflit entre le tyran et sa nièce. Les dramaturges insufflent de la nécessité dans un acte de courage pour le justifier et le valoriser. Cette idée de force supérieure qui incite à résister résonne dans Els camins de Antígona ${ }^{53}$ : la protagoniste déclare : «ce n'est plus Antigone qui choisit les chemins, à présent toutes les forces de la terre s'emparent d'elle. Elle ne résiste pas, elle les suivra jusqu'au bout. " ${ }^{54}$ Pendant la dictature franquiste, la mise en scène d'un individu que nul ne saurait résoudre au silence, face à un tyran qui refuse d'enterrer

51 M. Zambrano, op. cit. : "Antígona, en verdad, no se suicidó en su tumba, según Sófocles, incurriendo en un inevitable error, nos cuenta."

52 Ibid. : "Je suis fille de l'erreur".

53 A. Carrión, Els camins de Antígona, Buenos Aires, 1940.

54 Ibid., p. 69. 
dignement ses ennemis et qui impose à tous une obéissance sans faille ont nécessairement un écho très fort parmi le public. Non seulement parce que le contexte reflète la tragédie, mais surtout parce que cette figure et le message de la tragédie se trouvent actualisés par les artistes contemporains, grâce à l'universalité du symbole de résistance. En somme, comme l'écrit Rose Duroux, "alliant l'éthique à l'esthétique, Antigone serait à l'épreuve du temps. (...) Mais, augurent certains, de par sa surexposition même - qui vaut banalisation -, la figure mythique serait en passe de devenir un simple nom commun, synonyme de résistante. „ $\$ 5$

\author{
Fanny Blin \\ EA3656 AMERIBER \\ Université Bordeaux Montaigne \\ fanny.blin@live.fr
}

\begin{abstract}
Résumé
Immortalisée par la littérature engagée du $\mathrm{XX}^{\mathrm{e}}$ siècle en tant que symbole par excellence de l'opposition à la tyrannie, Antigone ressurgit particulièrement dans la dramaturgie espagnole pendant et après la dictature franquiste. Cette figure mythique a en effet inspiré près de vingt réécritures de l'œuvre sophocléenne entre 1939 et 1989, en castillan, mais aussi en catalan et en galicien. En effet, Antigone a hanté la pensée des opposants au Régime car sa tragédie familiale est miroir éloquent de la post-guerre civile. Dans un contexte où le pouvoir verrouille les discours historiographiques et contestataires, réécrire cette pièce offre un sillon à la pensée du drame national tout en valorisant l'objection de conscience. C'est le chemin qu'empruntent Bayo, Bergamín, Zambrano, Elizondo, De la Rica puis Riaza, dont les œuvres participent de la réinterprétation critique de la notion de désobéissance politique. Le rapport de force s'illustre dans l'écriture même : certains dramaturges catalans (Espriu, Muñoz i Pujol, Povill i Adserà...) et galiciens (Queizán, Lourenzo, et Rodríguez Pampín) combattent l'uniformisation culturelle menée par le Régime dictatorial, en transcrivant la tragédie dans une langue prohibée. Adapter cette pièce emblématique en langue régionale est donc en soi un acte de résistance. Mais à quoi résiste Antigone ? La figure fonctionne comme le masque des résistants : prêtant sa voix, elle ouvre la voie d'une possible contestation.
\end{abstract}

\title{
Mots-clés
}

Mythe, Antigone, dissidence, censure, antifranquisme, langues communautaires.

\begin{abstract}
Over the $\mathrm{XX}^{\text {th }}$ century, the European literary tradition immortalized Antigone's character as an archetypal symbol of the opposition to tyranny. In this context, the Greek heroin re-appeared frequently in Spanish drama, mostly during and after Franco's dictatorship. Indeed, this mythical character inspired nearly twenty rewritings of Sophocles' tragedy between 1939 and 1989, through Castilian voices, but also Catalan and Galician. Antigone haunted the thought of those who resisted the Regime because her family tragedy was a metaphor of the post-CivilWar period. As the power had locked any historiographical or critical discourse, rewriting this antic play was a possible way for Spanish playwrights to think and represent their national
\end{abstract}

55 R. Duroux et S. Urdician, op. cit., p. 31. 
drama while valuing political rebellion in fiction. Bayo, Bergamín, Zambrano, Elizondo, De la Rica and Riaza's versions show, through demystification, a contemporary interpretation of political disobedience. An ethical struggle is at stake, and some of the Spanish Antigones show provocation in the writing itself: several Catalan playwrights (like Espriu, Muñoz i Pujol, Povill i Adserà) and three Galicians (Queizán, Lourenzo, and Rodríguez Pampín) fight cultural standardization led by the dictatorial Regime, transcribing the tragedy in a prohibited language. Adapting this inherently resistant tragedy in a marginalized language is thus provocative, but Antigone, as a classical work, is like a mask for subversive messages.

\section{Keywords}

Myth, Antigone, censorship, antifrancoism, community languages. 